Monográfico «Contenidos educativos en abierto»

ARTÍCULO

Prácticas y recursos de educación abierta: la hoja de ruta OLCOS 2012

\author{
Guntram Geser
}

Fecha de presentación: febrero de 2007

Fecha de publicación: abril de 2007

\begin{abstract}
Resumen
En los últimos años, el movimiento Open Educational Resources u OER (recursos educativos abiertos o libres) ha atraído mucha atención debido, por ejemplo, a la amplia cobertura de los medios de comunicación sobre la iniciativa Open Courseware (software didáctico) del Instituto de Tecnología de Massachusetts, al trabajo de cada vez más organizaciones que promueven el uso de licencias Creative Commons y al éxito de sistemas basados en software Open Source (código abierto) como Moodle en el sector educativo. Sin embargo, para beneficiarse más de Open Educational Resources, es necesario entender mucho más claramente la función que OER puede desempeñar en el cambio de prácticas educativas. Por lo tanto, el proyecto Open e-Learning Content Observatory Services u OLCOS (servicios del observatorio de contenidos de aprendizaje virtual abierto), que es una acción transversal bajo el programa de e-learning europeo, ha producido una hoja de ruta con orientaciones y recomendaciones para los responsables de la toma de decisiones educativas sobre cómo fomentar todavía más el desarrollo y uso de OER. En este artículo se proporciona una breve visión general del contexto y el enfoque de la hoja de ruta OLCOS 20I 2, se explica por qué se da prioridad a prácticas educativas abiertas más que a recursos y se presentan algunos impulsores/facilitadores e inhibidores de prácticas y recursos de educación abierta. Además, resume algunas de las recomendaciones del informe de la hoja de ruta. El artículo también menciona y proporciona enlaces a cuarenta proyectos y recursos seleccionados que ilustran la riqueza y diversidad de las iniciativas actuales en educación abierta y prácticas y recursos relacionados.
\end{abstract}

Palabras clave

recursos de educación abierta, hoja de ruta, prácticas, políticas, recomendaciones

\title{
Open Educational Practices and Resources: The OLCOS Roadmap 2012
}

\section{Abstract}

In the last few years, Open Educational Resources (OER) have gained much attention; for example, due to the extensive media coverage on the Open Courseware initiative of the Massachusetts Institute of Technology, the work of ever more organisations that promote the use of Creative Commons licenses, and the success of Open Source software-based systems such as Moodle in the educational sector. However, in order to further benefit from Open Educational Resources it is necessary to gain a much clearer understanding of the role OER can play in changing educational practices. Therefore, the Open eLearning Content Observatory Services (OLCOS) project, which is a Transversal Action under the European eLearning Programme, has produced a roadmap to provide educational decision makers with orientation and recommendations on 
how to foster the further development and use of OER. This article provides a brief overview of the context and focus of the OLCOS roadmap $20 I 2$, explains why it gives priority to open educational practices rather than resources, and presents some drivers/enablers and inhibitors of open educational practices and resources. Furthermore, it summarises some of the recommendations of the roadmap report. The article also mentions and provides links to forty selected projects and resources that illustrate the richness and diversity of the current initiatives in open educational and related resources and practices.

\section{Keywords}

open educational resources, roadmap, practices, policies, recommendations

\section{Contexto y enfoque de la hoja de ruta $\mathrm{OLCOS} 2012$}

Se entiende que los recursos de educación abierta (OER) comprenden contenidos para enseñar y aprender, herramientas y servicios basados en software y licencias que permiten el desarrollo y la reutilización libre de contenidos, herramientas y servicios. La importancia de OER ha sido reconocida por la Unesco, la OCDE y otras organizaciones nacionales e internacionales que están interesadas en la creación e intercambio de tales recursos. Por ejemplo, el Centro de Investigación e Innovaciones Educativas (CERI) de la OCDE está llevando a cabo un estudio internacional sobre OER (que será completado a principios de 2007), y el Instituto Internacional de Planificación Educativa (IIEP) de la Unesco facilita una Comunidad de Interés en OER. Esa comunidad está en activo desde octubre de 2005 y tiene más de 600 miembros pertenecientes a 94 países.

En este contexto, el proyecto OLCOS ha elaborado una visión general de desarrollos actuales y probablemente futuros en OER mediante la presentación y evaluación de impulsores/facilitadores e inhibidores de prácticas y recursos de educación abierta. El objetivo ha sido identificar posibles logros en un horizonte cronológico definido para el año 20 I 2 y especificar cómo pueden abordarse los desafíos relacionados. El informe completo estará disponible a finales de enero del 2007 y se podrá descargar desde el sitio web del proyecto. ${ }^{\text {www }}$ Adicionalmente, el proyecto crea y pone a disposición un conjunto de paquetes de información, como tutoriales, ${ }^{\text {www }}$ y facilita el intercambio de conocimientos entre una comunidad de práctica europea en OER.
El consorcio del proyecto comprende el Centro $\mathrm{Eu}^{-}$ ropeo de Competencia en Medios (European Centre for Media Competence) (Alemania), La Red Europea de Educación Virtual y a Distancia (European Distance and E-Learning Network) (Hungría), la FernUniversität in Hagen (Alemania), el Grupo Mediamaisteri (Finlandia), la Universitat Oberta de Catalunya (España) y la entidad coordinadora del proyecto, el Salzburg Research / EduMedia Group (Austria).

El proyecto OLCOS considera que los recursos de educación abierta son un elemento importante de políticas que quieren aprovechar la educación y el aprendizaje permanente para la sociedad del conocimiento y la economía. No obstante, el proyecto también pone de relieve que para alcanzar ese objetivo es crucial promover la innovación y cambiar las prácticas educativas.

En particular, OLCOS advierte que la entrega de OER al todavía modelo dominante de transferencia de conocimiento centrada en el docente, tendrá poco efecto en cuanto a dotar a educadores, estudiantes y trabajadores de las aptitudes, conocimientos y habilidades necesarias para participar con éxito en la economía y sociedad del conocimiento.

Por lo tanto, la hoja de ruta resalta prácticas de educación abierta que se basan en un paradigma constructivista centrado en la competencia del aprendizaje y fomenta un compromiso creativo y de colaboración de estudiantes con contenidos, herramientas y servicios digitales en el proceso de aprendizaje. Sin embargo, se entiende que un cambio hacia tales prácticas sólo se producirá a más largo plazo en un proceso paso a paso. Llevar a cabo ese cambio requerirá esfuerzos concentrados y sostenidos de líderes educativos en todos los niveles. 


\section{Prioridad de las prácticas de educación abierta}

Muchos promotores de los recursos de educación abierta (OER) no tienen en cuenta el legado de entramados y modelos pedagógicos institucionales tradicionales. Parece que asumen implícitamente que el fácil y libre acceso a una «masa crítica de contenidos de gran valor» (que aparece como una fórmula estándar) y herramientas para usar tal contenido interactivamente, también conducirá de alguna manera a un cambio en esos entramados y modelos.

Los modelos pedagógicos a menudo ni se consideran en el debate de OER. Los motivos son múltiples: Por ejemplo, dado el objetivo de la Unesco de fomentar la libre disponibilidad de contenidos y herramientas de enseñanza y aprendizaje para el desarrollo de los países, el paradigma educativo sólo debe parecer de importancia secundaria. Otro motivo es que la discusión de OER ha estado dominada con frecuencia por consideraciones técnicas y administrativas en lugar de perspectivas de profesionales de la educación. Y un motivo más para la escasa comprensión de OER es el enfoque de muchos debates sobre cuestiones de los esquemas de licencia adecuados.

OLCOS promueve el acuerdo de que, antes de tratar contenidos abiertos útiles, herramientas y licencias, se deben considerar las prácticas pedagógicas sobre las que esos recursos pueden marcar la diferencia, es decir, usándolas como formas innovadoras de enseñanza y aprendizaje. Ello se debe a que, si el modelo dominante es la educación centrada en el docente (un docente arbitra el libro de texto o contenido del curso autorizado y los alumnos lo digieren y lo reproducen), los recursos de educación abierta no supondrán ninguna diferencia en la educación. En un modelo así, los docentes pueden descargar material docente de libre acceso en la web para preparar clases, y los estudiantes pueden usar algunos contenidos para preparar el material de las lecciones, pero seguirá siendo un canal unidireccional de provisión de contenidos, en el que el libro de texto físico o el curso son sustituidos por material digital.

Profesores y alumnos seguirán siendo consumidores de contenido prefabricado, sin llegar a ser creativos y colaborar por sí mismos, y no "pagarán con la misma moneda» aportando sus propios contenidos o añadiendo valor al contenido de otros (por ejemplo, material mejorado, casos prácticos, lecciones aprendidas, etc.). Por lo tanto, la hoja de ruta de OLCOS también fomenta un cambio en la función profesional, el conocimiento propio, las actitudes y las habilidades de los profesores. Eso podría, por ejemplo, incluir el cuestionamiento, la evaluación y la mejora permanente de prácticas y recursos educativos, y el intercambio, dentro de una comunidad de práctica, de experiencias, lecciones aprendidas y sugerencias sobre cómo promover mejor el desarrollo de los estudiantes y de las propias capacidades y habilidades. Esas contribuciones pueden ser los recursos más importantes de valor añadido de OER.

\section{Impulsores/facilitadores e inhibidores importantes de prácticas y recursos de educación abierta}

En la hoja de ruta de OLCOS se tratan las siguientes áreas: políticas, estructuras institucionales y modelos empresariales, repositorios de acceso libre y contenido abierto, y laboratorios de prácticas y recursos de educación abierta. Para cada una de esas áreas se identifican impulsores/facilitadores e inhibidores de prácticas y recursos de educación abierta, y se describen con detalle. Los resultados se resumen en los Extractos de la hoja de ruta, que se pueden emplear como puntos de arranque para debatir iniciativas en OER y prácticas de educación abierta en un nivel estratégico.

En este artículo sólo se puede presentar el resumen de algunos de los más importantes impulsores/facilitadores e inhibidores de prácticas y recursos de educación abierta. A continuación se abordan bajo los encabezados siguientes: políticas educativas, modelos de negocio, estructuras institucionales, repositorios de contenidos educativos y comunidades de práctica, y nuevas herramientas en escena.

\section{I. Políticas educativas}

A pesar de las grandes inversiones en infraestructuras de e-learning de las instituciones educativas (hardware/software, conectividad, sistemas de gestión de aprendizaje, etc.), durante los últimos diez años se ha conseguido poco impacto en lo que a cambiar prácticas educativas se refiere. Por consiguiente, la política educativa exige cada vez más un fuerte compromiso de directores, administradores y personal de las instituciones educativas sobre la innovación en la educación y el cambio organizativo.

Hay una preocupación creciente de que las instituciones educativas no apoyen eficazmente a los estudiantes para que adquieran las capacidades $y$ habilidades necesarias para participar con éxito en la sociedad y la economía del 
conocimiento. Existe un problema apremiante respecto a la idoneidad de los programas de aprendizaje permanente para asegurar competitividad económica y empleabilidad de trabajadores para trabajos de valor superior (industrias basadas en el conocimiento).

Por ello, particularmente en el área de aprendizaje permanente basado en TIC podemos esperar una comprensión cada vez mayor de la importancia de OER para impulsar la participación. Un ejemplo de esto son los recientes experimentos de algunas universidades de educación a distancia o abierta al ofrecer cursos abiertos de aprendizaje autodidacta con el objetivo de «convertir» a los usuarios en estudiantes inscritos (esos proyectos los lleva a cabo actualmente la Asociación Europea de Universidades de Educación a Distancia, la Universidad Abierta de Holanda y la Universidad Abierta del Reino Unido). El escenario de este experimento es la competición global en educación superior y la previsible disminución en número de estudiantes en Europa debido a las tendencias demográficas.

\subsection{Modelos de negocio}

Actualmente existe un nivel saludable de competencia entre instituciones líderes para proporcionar acceso libre a recursos educativos. Muchas iniciativas empezaron después de la extensa cobertura mediática del proyecto Open Courseware del MIT, que fue anunciado en abril del $200 \mathrm{I}$. Por ejemplo, en la segunda mitad del 2006 el OpenCourseWare Consortium (Consorcio de Software Didáctico Abierto) internacional tenía más de roo miembros.

Sin embargo, los proyectos más grandes y conocidos están financiados de forma importante, por lo que los modelos de negocio para iniciativas OER sostenibles son un tema de preocupación. De hecho, los modelos de negocio en OER son complicados y se debe hallar la combinación correcta de flujos de ingresos (por ejemplo, financiación pública y/o privada, patrocinio, donaciones, servicios de pago). Esto se hará todavía más difícil porque aumentará la competencia por los escasos recursos financieros (también en las instituciones).

Además, aunque actualmente vemos mucha provisión de software didáctico estático (con más frecuencia en formatos cerrados), «los recién llegados» al movimiento OER tendrán que convencerse mediante recursos muy útiles y usuarios activos que estén dispuestos a compartir abiertamente material educativo propio.

También se ha de destacar que las editoriales de temas educativos consideran el movimiento OER como una amenaza para sus intereses comerciales, lo que hará difícil establecer asociaciones privadas-públicas innovadoras relacionadas con OER. En los años venideros habrá posiblemente una brecha cada vez más amplia entre el contenido didáctico tradicional que está protegido por tecnologías de administración de derechos digitales y la mayor circulación de contenido que se comparta libremente (por ejemplo, basado en licencias Creative Commons).

\subsection{Estructuras institucionales}

La cultura establecida en instituciones académicas y de educación superior no fomenta especialmente la creación, intercambio y reutilización de recursos de educación abierta. En las universidades se suele dar más valor a la investigación que a la docencia, en particular cuando se trata de promoción académica. Por lo tanto, normalmente existe poco incentivo y apoyo para que los profesores experimenten con innovadoras formas mejoradas de TI para la enseñanza y para sobresalir en la producción e intercambio de material educativo.

De hecho, los expertos tienen un amplio consenso en que las recompensas institucionales adecuadas (por ejemplo, relevancia significativa en promoción académica $u$ otras) son el factor más importante para que haya iniciativas OER con éxito por parte de instituciones académicas y educativas. Las motivaciones altruistas o la posibilidad de «ganar reputación» pueden no ser impulsores suficientemente fuertes como para invertir el tiempo y esfuerzo necesario para crear OER más allá del tipo software didáctico, como apuntes de clase y listas de lectura.

No obstante, también hay otros dos problemas importantes en OER desde la perspectiva institucional: en primer lugar, a menudo hay una carencia de regulaciones bien definidas en relación con derechos de propiedad intelectual/copyright; en segundo lugar, las iniciativas OER, que buscan promover la creación e intercambio de OER entre profesores, deberán invertir un considerable esfuerzo en formación y soporte técnico.

\subsection{Repositorios de contenido educativo y comunidades de práctica}

Durante los últimos diez años, se ha desarrollado un sólido know-how sobre cómo hacer accesible información en repositorios distribuidos, y también hacer posible la búsqueda combinada (federated search). Eso incluye hacer uso 
del método Open Archive Initiative (iniciativa de archivos abiertos) basado en su Protocol for Metadata Harvesting (protocolo para recogida de metadatos), respositorios $\mathrm{P}_{2} \mathrm{P}$ y/o implementaciones creadas sobre SQI (interfaz de consulta simple) para búsqueda combinada a través de repositorios de objetos de aprendizaje.

Pero en este momento hay poca experiencia sobre cómo apoyar eficazmente a comunidades de práctica mediante repositorios educativos. Las iniciativas educativas, especialmente las nacionales más grandes, todavía siguen una estrategia de arriba abajo que intenta entregar una «masa crítica» de objetos de aprendizaje para una educación centrada en el profesor. Lo que a menudo no se entiende es que este modo de prestación refuerza el paradigma centrado en el profesor sobre la educación, todavía dominante, y se opone al objetivo de las prácticas de enseñanza y aprendizaje innovadoras.

Para que las prácticas educativas innovadoras emerjan y florezcan, debe permitirse a profesores y estudiantes que sean creativos y compartan recursos que hallen útiles en ciertos contextos de aprendizaje. Por lo tanto, los repositorios educativos tendrán que pensar más atentamente acerca de cómo resultar útiles a las comunidades de práctica, lo que es de la mayor importancia si las iniciativas OER quieren crecer basándose en contribuciones de los usuarios y en el intercambio de contenido entre ellos.

Normalmente, un modelo de proveedor que se establezca para hacer algo para comunidades de práctica - a menudo para proporcionar acceso a una base de datos de contenido- no funcionará. Más bien, tales comunidades deben ser habilitadas para hacer y lograr algo por sí mismas. De hecho, la noción de una comunidad de práctica implica que los miembros de esa comunidad comparten un interés en promocionar prácticas concretas y quieren desarrollar más know-how, tanto para abordar ciertos problemas como para recursos tales como el contenido educativo. Para apoyar a las comunidades de práctica, los repositorios educativos deberán implementar «nuevas herramientas en escena» disponibles.

\subsection{Nuevas herramientas en escena}

Los últimos años han visto un aumento enorme del uso de herramientas de software social y servicios como weblogs, wikis, redes sociales, intercambio de marcadores y contenidos, etc., más allá del sector educativo. Como esta nueva generación de herramientas y servicios basados en web faculta a los estudiantes a crear y compartir fácilmente conte- nidos, incluso el más pequeño «rebosamiento» puede tener un impacto considerable en cuanto a cambios en prácticas educativas. Sin embargo, actualmente el uso de software social por parte de profesores individuales y organizaciones docentes está en fase experimental.

En la actualidad, el entorno web está cambiando drásticamente y los contenidos digitales han llegado a ser muy fluidos. Puede producirse, sindicarse, montarse y empaquetarse más fácilmente de distintas formas. Además, los servicios que proporcionan algún tipo de información se pueden combinar para ofrecer nuevas y asombrosas maneras de integrar contenidos (también llamados «mashups»).

Otra base importante de la explosión de servicios es el mecanismo de suministro web Really Simple Syndication (RSS), que se ha convertido en un estándar para distribución de contenidos y sindicación. Eso puede ser empleado por proveedores de acceso a contenido educativo para aportar información fresca y continuamente actualizada a sus portales. De particular interés será permitir a los estudiantes individuales y grupos de estudio seleccionar suministros sobre ciertos temas que les brinden contenidos de relevancia temática, incluyendo podcasts (audio) y videocasts. Sin embargo, los suministros RSS no necesariamente tienen que llevar una etiqueta educativa. Más bien, los estudiantes que se interesen en una cuestión de investigación determinada a menudo ganarán más suscribiéndose a suministros de instituciones no gubernamentales, organizaciones científicas, servicios de información empresarial o secciones de servicios de noticias internacionales.

Para los repositorios educativos también será esencial conectar más eficazmente a profesores y alumnos con el cuerpo de conocimientos codificados de ciertos dominios, por ejemplo tesauros, sistemas de clasificación, ontología de dominios. Se espera que durante los próximos cinco a diez años las aplicaciones de web semántica proporcionarán formas novedosas de hacer uso de tales recursos de conocimiento. Por ejemplo, ya hay interesantes ejemplos de acceso basado en conceptos, aplicaciones de filtro semántico y exploración, y herramientas de software social como wikis semánticos.

\section{Recomendaciones para las partes interesadas}

El informe de la hoja de ruta de OLCOS proporciona un conjunto completo de recomendaciones para las partes interesadas, desde responsables de política educativa y 
organismos de financiación a profesores y estudiantes particulares. Entre las acciones sugeridas están las siguientes:

Los diseñadores de políticas educativas y organismos de financiación deben exigir que los recursos académicos $y$ educativos que han sido totalmente o en gran parte financiados públicamente se hagan libremente accesibles bajo una licencia apropiada (como Creative Commons o similar). Por ejemplo, las licencias para contenido educativo deberían estar idealmente libres de restricciones para modificar, combinar y readaptar el contenido.

Respecto a repositorios educativos de acceso abierto, los organismos de financiación deberían concentrarse en fomentar el desarrollo de repositorios ampliamente usados, tecnológicamente de vanguardia y sostenibles. Los criterios de selección de proyectos deberían exigir que los proponentes muestren un profundo conocimiento de cómo establecer el uso activo del repositorio tan ampliamente como sea posible. Los esquemas de financiación deberían proporcionar una perspectiva a más largo plazo, mediante la financiación inicial, para lograr el pleno funcionamiento y financiación adicional basada en una evaluación crítica del uso real.

Se recomienda a los consejos, directores y supervisores de instituciones educativas que examinen si éstas emplean enfoques innovadores más allá de la transferencia clásica de conocimientos centrada en el profesor. Por ejemplo, deberían preguntar a las instituciones educativas qué cantidad de trabajo de los profesores se concentra en preparar a los estudiantes para identificar problemas del mundo real, aclarar métodos de estudio, evaluar la relevancia de informaciones y observaciones, y debatir críticamente los resultados de estudio.

En relación con el intercambio y la reutilización de recursos abiertos de un fondo común de contenidos, herramientas y servicios, la hoja de ruta sugiere el establecimiento de cooperaciones formales entre organizaciones educativas. Entre los efectos positivos no sólo se consideran las reducciones de costes en el desarrollo y gestión de recursos, sino también el aprovechamiento de su calidad. Por ejemplo, el hecho de que los recursos que se hayan hecho disponibles serán evaluados de forma crítica por instituciones asociadas también tendrá un impacto favorable sobre los criterios y el control de calidad interna.

En las iniciativas de recursos de educación abierta, los mecanismos de premio y las medidas de apoyo se consideran muy importantes para impulsar el desarrollo e intercambio de recursos en el nivel institucional. Los consejos, directores y supervisores tendrán que cuestionar valores, tradiciones y prácticas establecidas: por ejemplo, el mayor valor que se suele conceder a la investigación en compara- ción con la docencia, particularmente cuando se trata de promoción académica.

La hoja de ruta también subraya que en muchas instituciones dista de estar claro quién posee los derechos de propiedad intelectual/copyright y qué licencias se deben emplear cuando los recursos se ponen a disposición de otros. Sobre ello se recomienda que los contratos de investigadores y educadores empleados reconozcan los derechos de propiedad intelectual de los autores, pero también requieran derechos de reproducción no exclusivos para que la institución haga accesibles los recursos educativos bajo las licencias apropiadas. Además, se deben implementar mecanismos que (semi) automáticamente adjunten licencias al material que se pone a disposición libre.

Respecto al conjunto de recomendaciones para los estudiantes, puede resultar interesante que la hoja de ruta invite a los estudiantes a retar a los profesores con solicitudes como: ¿por qué no usar weblogs para compartir ideas, observaciones y enlaces comentados a material de estudio útil? ¿Por qué no usar un wiki para un proyecto de estudio en colaboración? ¿Por qué no suscribirse a suministros RSS temáticos que proporcionan a un proyecto la información del «mundo real» relevante y regularmente actualizada? Una recomendación adicional para los estudiantes es que tengan un e-portfolio (cartera electrónica) para documentar y reflejar el avance y los resultados de su trabajo de estudio, y para poner a disposición de los demás los resultados de los que están orgullosos a través de un repositorio de acceso libre bajo una licencia de contenido abierto.

\section{Proyectos y recursos seleccionados}

Los cuarenta proyectos y recursos siguientes se han seleccionado para ilustrar la riqueza y diversidad de las iniciativas actuales en educación abierta y recursos y prácticas relacionados:

AVOIR - Iniciativas y recursos de educación abierta virtual africana

$<$ http://avoir.uwc.ac.za>

Centro de aprendizaje abierto y sostenible (COSL) / Conferencias Educación Abierta

$<\mathrm{http} / / /$ cosl.usu.edu>

Commonwealth of Learning - Learning Object Repository (Mancomunidad de Aprendizaje - Repositorio de objetos de aprendizaje) <http://www.col.org/ colweb/site/pid/2922> 
Connexions (Conexiones) (plataforma en línea para la gestión e intercambio de módulos de cursos abiertos) <http://cnx.org>

Creative Commons $<$ http://creativecommons.org>

Development Gateway - Recursos de educación abierta (tiene como objetivo poner en práctica Internet para los países en desarrollo)

$<$ http://topics.developmentgateway.org/openeducation>

Directory of Open Access Journals (Directorio de publicaciones de libre acceso) $<$ http://www.doaj.org>

Edublogs Awards (Premios a blogs educativos) <http://www.incsub.org/awards>

EducaNext (servicio de intermediación de contenido abierto para educación superior) <http://www.educanext.org>

Education Podcast Network (Red de podcasts educativos) $<$ http://epnweb.org>

Elgg.net (iniciativa de software de la comunidad educativa) <http://elgg.net>

Freesound (creciente base de datos de sonidos con licencia Sampling Plus de Creative Commons) $<$ http://freesound.iua.upf.edu $>$

GLEF Learning Interchange \& Edutopia (George Lucas Educational Foundation) $<$ http://ali.apple.com/ali_sites/glefli >

Global SchoolNet Foundation (fomenta la cooperación internacional en aprendizaje basado en problemas/ proyectos) <http://www.globalschoolnet.org>

Proyecto GlobalText (su objetivo es crear una biblioteca gratuita con r.ooo libros de texto electrónicos para estudiantes del mundo en desarrollo) $<$ http://globaltext.org>

GLOBE - Global Learning Objects Brokered Exchange

GLOBE - Intercambio con mediación de objetos de aprendizaje global (colaboración de Ariadne, Education.au, eduSource Canadá, MERLOT y NIME) <http://globe.edna.edu.au/globe/go>

Proyecto INDICARE (diálogo informado sobre aceptación del consumidor de soluciones de gestión de derechos digitales en Europa) <http://www.indicare.org>

iRights.info (recurso de información sobre derechos de propiedad intelectual y copyright; información en alemán) <http://irights.info >
Lernmodule.net (repositorio de contenido abierto para el sector académico en Alemania) $<$ http://lernmodule.net>

LibriVox (fomenta audiolibros gratuitos de dominio público)

$<$ http://librivox.org>

M.I.T. Open Courseware (OCW)

Software didáctico abierto (OCW) del M.I.T

$<$ http://ocw.mit.edu>

MathWorld (amplio recurso de matemáticas gratuito) $<$ http://mathworld.wolfram.com>

OCDE - Centro de investigación e innovación educativa (CERI): Proyecto/encuesta de recursos de educación abierta

<http://www.oecd.org/document/20/o,2340,en_ 2649_3584558 I_35023444_I_I_I_I,oo.html>

Open Education Association Asociación de educación abierta (fomenta el concepto de OER entre profesores de universidades e institutos superiores) <http://www.openeducationassociation.org>

Open Educator (se centra en intercambio de conocimientos, herramientas y recursos en OSS) < http://www. openeducator.org>

Open Knowledge Network Red de conocimiento abierto (fomenta la recopilación e intercambio de conocimiento local usando soluciones técnicas flexibles; opera en África, Sudeste Asiático y Latinoamérica) <http://www.openknowledge.net>

OpenCourse.org ( $«$ Contenido abierto + Comunidad $=$ Curso abierto»)

<http://www.OpenCourse.org>

OpenDOAR - Directory of Open Access Repositories OpenDOAR - Directorio de repositorios de acceso libre $<$ http://www.opendoar.org>

OpenLearn - Universidad Abierta del Reino Unido $<$ http://openlearn.open.ac.uk>

Proyecto Gutenberg <http://www.gutenberg.org>

Public Knowledge Project

Proyecto de conocimiento público (desarrolla software de código abierto gratuito para la gestión, publicación e indexación de revistas y conferencias) <http://www.pkp.ubc.ca>

Reading and Writing for Critical Thinking Internacional Consortium (Lectura y escritura para el Consorcio internacional de pensamiento crítico) (con el apoyo de la Red de Fundaciones Soros)

$<$ http://ct-net.net> 
Schoolforge (su objetivo es que las escuelas disfruten de las ventajas del software de código abierto y gratuito) $<$ http://www.schoolforge.net>

Science Commons (su objetivo es eliminar barreras en el flujo de conocimiento científico e información técnica) $<$ http://sciencecommons.org>

Encuesta de proyectos de contenido abierto en países no occidentales

$<$ http://oc.openflows.org

Textbook Revolution (base de datos de libros de texto que se puede consultar) <http://textbookrevolution.org>

UNESCO - IIEP Comunidad de interés en recursos de educación abierta / recursos útiles de OER <http://oerwiki.iiep-unesco.org>

Portal de software de código abierto y gratuito de la UNESCO

<http://www.unesco.org/cgi-bin/webworld/portal_ freesoftware/cgi/page.cgi? $\mathrm{d}=\mathrm{I}>$

Wikilibros (colección de libros de texto de contenido abierto basados en Wiki) <http://en.wikibooks.org>

WikiEducator (iniciativa de Commonwealth of Learning para desarrollar recursos educativos gratuitos en línea) $<$ http://www.wikieducator.org>

Banco Mundial - Youthink! <http://youthink.worldbank.org>

\section{Bibliografía}

A continuación ofrecemos una selección de algunas publicaciones relevantes en general de la bibliografía de la hoja de ruta en la que se enumeran 250 publicaciones:

ALBRIGHT, PAUL (2005). «Final forum report». En: Internet Discussion Forum: Open Educational Resources. Open Content for Higher Education (24 de octubre - 2 de diciembre de 2005). [Informe en línea]. UNESCO - Instituto Internacional de Planificación Educativa. [Fecha de consulta: I 2 de diciembre de 2006]. <http://www.ifap.ru/library/booko64.pdf>

ALEXANDER, BRYAN (2006). «Web 2.0. A new wave of innovation for teaching and learning?» [artículo en línea]. Educause Review. Marzo/abril 2006. [Fecha de consulta: I 2 de diciembre de 2006].

<http://www.educause.edu/ir/library/pdf/ermo62 I.pdf>

BATEMAN, PETER; TUCKER, KIM (2006). «Report of the discussions on developing a research agenda for Open
Educational Resources». En: International Community of Interest. Open Educational Resources. Open Content for Higher Education. [Informe en línea]. UNESCO - Instituto Internacional de Planificación Educativa. [Fecha de consulta: I 2 de diciembre de 2006]. <http://oerwiki.iiep-unesco.org/images/f/fa/ OER_research_agenda_report.pdf>

Bechler, rosemary (2006). Unbounded Freedom. A guide to Creative Commons thinking for cultural organisations [libro en línea]. Londres: Counterpoint. [Fecha de consulta: I 2 de diciembre de 2006].

<http://www.counterpoint-online.org/download/ 325 /Unbounded-freedom.pdf>

COMISIÓN EUROPEA. DIRECCIÓN GENERAL DE EDUCACIÓN Y Cultura (noviembre de 2004). «Key Competences for Lifelong Learning: a European Reference Framework». En: Implementation of the "Education and Training» 2010 Work Programme. Working Group B "Key Competences». [Fecha de consulta: I 2 de diciembre de 2006].

<http://europa.eu.int/comm/education/policies/ 2010/doc/basicframe.pdf>

DANIEL, JOHN; WEST, PAUL; MACKINTOSH, WAYNE (2006). «Exploring the role of ICTs in addressing educational needs: identifying the myths and the miracles». En: NADEOSA roth Anniversary Conference. Pretoria, Sudáfrica [artículo en línea].

<http://www.col.org/colweb/site/pid/4042>

Downes, stephen (2006). Models for Sustainable Open Educational Resources. En: Reunión de expertos de la $O C D E$ sobre recursos de educación abierta (6-7 de febrero de 2006: Malmö, Suecia). [Fecha de consulta: I 2 de diciembre de 2006].

<http://www.oecd.org/dataoecd/3/5/3678 I 698.pdf> HOLMES, BRIAN (2005). «E-learning content - a European policy perspective». En: Open Culture: Accessing and Sharing Knowledge Workshop (27-29 de junio de 2005: Universidad de Milán). [Fecha de consulta: I 2 de diciembre de 2006].

<http://www.aepic.it/conf/viewpaper.php?id= Io6\&cf $=3>$

HYLÉN, JAN (2006). «Open Educational Resources: Opportunities and Challenges». En: Open Education 2006: Community, Culture, and Content (27-29 de septiembre de 2006: Utah State University, Logan, UT). [Actas finales en línea] [Fecha de consulta: I 2 de diciembre de 2006].

<http://cosl.usu.edu/conferences/opened 2006/ proceedings $>$ 
OCDE - Centro de investigación e innovación educativa (2006). «Notes from Expert Meeting on Open Educational Resources». (6-7 de febrero de 2006: Malmö, Suecia). [Fecha de consulta: I 2 de diciembre de 2006]. <http://www.oecd.org/dataoecd/ I 2/46/36 I62 I 43.pdf> OWEN, M.; GRANT, L.; SAYERS, s.; FACER, K. (2006). Opening Education: Social software and learning [informe en línea]. Bristol: Futurelab. [Fecha de consulta: I 2 de diciembre de 2006].

<http://www.futurelab.org.uk/download/pdfs/research/ opening_education/Social_Software_report.pdf>

WILEY, DAVID (septiembre de 2006). «On the Sustainability of Open Educational Resource Initiatives in Higher Education» [artículo en línea]. Utah State University:
Center for Open and Sustainable Learning. [Fecha de consulta: I 2 de diciembre de 2006].

$<$ http://opencontent.org/docs/oecd-report-wileyfall-2006.pdf>

willinsky, john (2006). The Access Principle: The Case for Open Access to Research and Scholarship [libro en línea]. Cambridge y Londres: MIT Press. [Fecha de consulta: I 2 de diciembre de 2006].

<https://mitpress.mit.edu/books/willinsky/ TheAccessPrinciple_TheMITPress_026223242 I.pdf>

\section{Cita recomendada}

GESER, GUNTRAM (2007). «Prácticas y recursos de educación abierta: la hoja de ruta OLCOS 20I2». En: «Contenidos educativos en abierto» [monográfico en línea]. Revista de Universidad y Sociedad del Conocimiento (RUSC). Vol. 4, n. ${ }^{\circ}$ I. UOC. [Fecha de consulta: $\mathrm{dd} / \mathrm{mm} / \mathrm{aa}]$. $\langle$ http://www.uoc.edu/rusc/4/r/dt/esp/geser.pdf > ISSN I698-580X

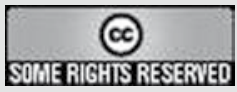

Los textos publicados en esta revista están sujetos - si no se indica lo contrario- a una licencia de Reconocimiento 3.0 España de Creative Commons. Puede copiarlos, distribuirlos, comunicarlos públicamente y hacer obras derivadas siempre que reconozca los créditos de las obras (autoría, nombre de la revista, institución editora) de la manera especificada por los autores o por la revista. La licencia completa se puede consultar en http://creativecommons.org/ licenses/by/3.0/es/deed.es. 


\section{Sobre el autor}

Guntram Geser

Jefe de Investigación de la Sociedad de la Información en Salzburg Research Forschungsgesellschaft m.b.H. guntram.geser@salzburgresearch.at

Guntram Geser dirige el Departamento de Investigación de la Sociedad de la Información de Salzburg Research Forschungsgesellschaft m.b.H. de Austria. Tiene amplia experiencia en proyectos financiados por la UE (prioridad en tecnologías de la sociedad de la información o IST, y otros programas) y en proyectos nacionales tal como se llevan a cabo en centros de competencia industrial. Las tareas principales en esos proyectos son el desarrollo de hojas de ruta de investigación y desarrollo de tecnologías de información y comunicaciones, monitorización y evaluación de tecnologías y estudios sobre la adopción, uso e impacto de tecnologías de información y comunicaciones (TIC). Hay proyectos ejemplares como EPOCH - Excellence in Processing Open Heritage (Red de excelencia FP6IST, 2004-2008), http://www.epoch-net.org; DigiCULT Forum (Medida complementaria FP 5 -IST, 2002-2004), http://www.digicult.info; EP 2010 - Futuro de la publicación electrónica en el 2010 (estudio estratégico para la Comisión Europea, DG Information Society, Dirección E, 2002/2003), http://ep2oro.salzburgresearch.at. Guntram Geser tiene un doctorado (Ciencias Políticas y de Comunicación) de la Universidad Paris Lodron, Salzburgo, y un máster de Estudios avanzados en gestión telemática de la Universidad de Donau, Krems, Austria. Antes de unirse a la investigación de Salzburgo, trabajó en estudios culturales de tecnología y medios en proyectos realizados en Berlín (Universidad Técnica de Berlín, Deutsche Film- und Fernsehakademie) y Amsterdam (Instituut for Film- en Televisiewetenschap, Nederlands Filmmuseum). También participó en la Unidad de Investigación Interdisciplinaria de Viena para el estudio de ciencias técnicas y sociedad, y trabajó como asesor de medios para el Servicio Cultural Austriaco. 